\title{
Helix Sense-Selective Polymerization of Chloral with Chiral Alkoxide Initiators: Tuning the Chiroptical Properties of the Polymer
}

\author{
Gary D. JAYCOX ${ }^{* \dagger}$ and Otto $\operatorname{VoGL}^{\dagger \dagger}$ \\ Department of Polymer Science and Engineering, Materials Research Laboratory, \\ University of Massachusetts, Amherst, MA 01003, U.S.A.
}

(Received April 15, 1991)

\begin{abstract}
The helix sense-selective polymerization of chloral with chiral lithium alkoxides derived from $R(-), S(+)$-2-octanol and four mono-hydroxy sterols afforded optically active polymer samples with high solid state optical rotations at the sodium D-line $(589 \mathrm{~nm})$. This chiroptical behavior stemmed from a predominance of a single screw direction along the polymer's rigid helical backbone. The sign and magnitude of the optical rotation enhancements were found to depend upon both initiator structure and the conditions under which the polymerizations were carried out. By carefully manipulating experimental conditions, polychloral samples with specific rotations ranging from $(+) 4000^{\circ}$ to $(-) 4000^{\circ}$ were readily prepared. Model end-group studies confirmed that the presence of asymmetric initiator fragments in the polymer contributed little to the observed optical rotations reported here. Polychloral prepared with achiral or racemic initiators was optically inactive.
\end{abstract}

KEY WORDS Asymmetric Polymerization / Chiral Initiators / Helical Asymmetry / Optical Activity /

Synthetically derived polymers that possess ordered or well-defined molecular architectures comprise an interesting and rapidly expanding class of materials. Notable advances in this arena have led to macromolecules with unusual helical, branched, combed, and star-like orientations. $^{1-4}$ Not unexpectedly, the physiochemical and biological properties of these novel derivatives often differ fundamentally from those of their more randomly ordered counterparts.

Over the past decade, efforts in our laboratory have focused on the synthesis and chiroptical evaluation of helically asymmetric polyaldehydes. We have placed particular emphasis on those derivatives that maintain their unique geometries in the absence of main chain or pendent side chain chiral centers. As reviewed earlier, ${ }^{5}$ optically active polymers of this type can be prepared with chiral initiators which asymmetrically induce the formation of a single screw direction along a growing polymer chain. When applied to the polymerization of chloral (trichloroacetaldehyde), the asymmetric or helix sense-selective polymerization technique has afforded polymer samples with exceptionally high optical rotations at the sodium D-line. ${ }^{6-8}$ Similar studies have been reported for poly(triphenylmethyl methacrylate $)^{9,10}$ and for several polyisocyanides. ${ }^{11,12}$

The preparation of optically active poly-

* To whom correspondence should be addressed.

† Present address: Central Research and Development Department, E.I. du Pont de Nemours and Company, Experimental Station, Wilmington, DE 19880, U.S.A.

${ }^{\dagger \dagger}$ Present address: Polytechnic University, Brooklyn, NY 11201, U.S.A. 
mers with asymmetry soley at the helical or macromolecular level requires that the polymer chain form a tight, rigid helix with no conformational reversals. Crystallographic ${ }^{13}$ and spectroscopic data ${ }^{14-16}$ indicate that polychloral is both isotactic and characterized by stable $4_{1}$ helical order in its solid state. Steric conflicts centered between adjacent trichloromethyl side groups aid in stabilizing the persistence of one screw direction along the polyaldehyde chain. Of added concern, however, have been the initiation and early propagation steps that ultimately lead to the formation of the polymer helix. Chiral initiators will induce the formation of a single screw sense only if each step occurs with a high degree of stereoselectivity. Model studies in our laboratory employing a series of acetatecapped chloral oligomers have recently confirmed that oligomeric chain growth proceeds in a highly stereoselective, isotactic fashion, with the embryonic stages of helix development well underway be the third chloral addition step. ${ }^{17-22}$ In light of these and other findings, polychloral appears to be well suited for studies of this type.

Herein, we report that lithium alkoxides $\mathbf{1}-\mathbf{5}$ derived from commercially available secondary alcohols are capable of imparting exceptionally
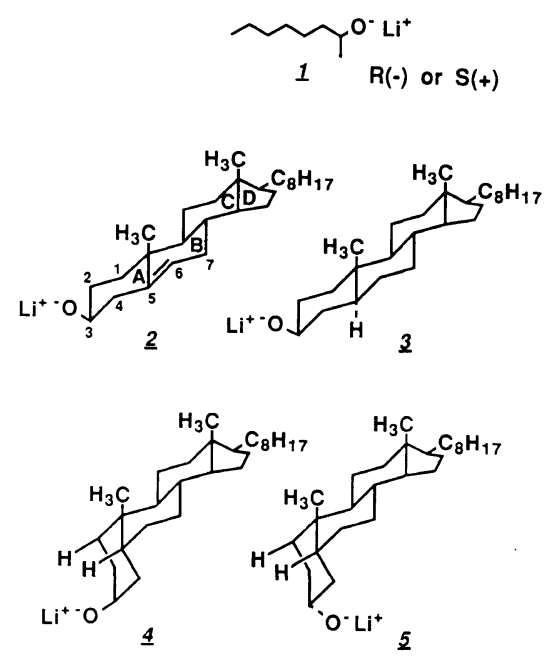

large solid state optical rotations into polychloral. Moreover, with the proper choice of initiator, and with the controlled manipulation of the polymerization reaction, specific rotations in the polymer can be adjusted or tuned from (+) $4000^{\circ}$ to $(-) 4000^{\circ}$ (sodium D-line), allowing for the preparation of a polymeric solid with tailor-made chiroptical properties. The full details of these efforts are provided below. ${ }^{23,24}$

\section{EXPERIMENTAL}

\section{Materials}

$R(-)-, S(+)-2-$ Octanol (Fluka, optical purities $>98 \%$ ) and rac-2-octanol (Aldrich) were used as received. Sterols (Pfaltz and Bauer) were twice recrystallized from warm absolute ethanol and then dried in vacuo at room temperature. $n$-Butyllithium (Alfa-Ventron, $2.1 \mathrm{M}$ in hexanes) was standardized with $2,5-$ dimethoxybenzyl alcohol before use. ${ }^{25} \mathrm{Li}$ thium-t-butoxide (Alfa-Ventron) was purified by sublimation. Chloral (Montrose) was fractionally distilled from $\mathrm{P}_{2} \mathrm{O}_{5}$ under an inert atmosphere in a manner described earlier. ${ }^{26}$ Purity of monomer grade chloral was $>99.9 \%$ as judged by gas chromatography. $n$-Hexane (Aldrich) was fractionally distilled from sodium wire. Diphenyl ether, acetic anhydride, methanol and absolute ethanol (Aldrich) were all reagent grade materials.

\section{Polymerization of Chloral with Chiral Alkoxides (Method I)}

Initiators $\mathbf{1}-\mathbf{5}$ were first prepared by treating the corresponding alcohol $(1.0 \mathrm{mmol})$ in hexane $\left(1.0 \mathrm{ml}, 0^{\circ} \mathrm{C}\right)$ with 0.98 equiv. of $n$-butyllithium under dry nitrogen. Freshly distilled chloral $(39.0 \mathrm{ml}, 400 \mathrm{mmol})$ was added to a desired alkoxide and the initiated monomer solution that resulted was held isothermally at 65,75 , 85 or $95^{\circ} \mathrm{C}$ under nitrogen for $10 \mathrm{~min}$ before being syringed into glass sheet-casting assemblies (described below). On rapidly cooling the loaded assemblies to $0^{\circ} \mathrm{C}$, the solutions were 
cryotachensically polymerized ${ }^{27}$ in sheet form. After $12 \mathrm{~h}$, the resulting sheets were gently removed from their casting units and subsequently treated with acidic methanol $(10 \%$ $\mathrm{HCl})$ to protonate dormant alkoxide endgroups trapped in the polymer. The polychloral sheets were trimmed into circular disks, each with a diameter of $1.0 \mathrm{~cm}$. The translucent disks were then soaked in diphenyl ether, a refractive index-matching medium, until optically transparent. Care was taken to insure that the chiroptical properties of the polymer samples were not altered during the work-up procedure. Sample thickness as carefully determined by a friction-stop micrometer was of the order of $0.1 \mathrm{~mm}$.

\section{Polymerization of Chloral (Method In)}

Polymerizations were carried out for initiators $\mathbf{1}-\mathbf{5}$ as described in method I above except that the time between initiation and polymerization events was varied from 2.5 to $120 \mathrm{~min}$. For longer incubation periods, holding temperatures were typically $\pm 1^{\circ} \mathrm{C}$.

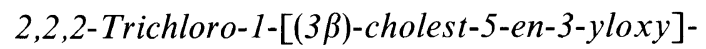 ethyl acetate (6)}

Under dry nitrogen, $n$-butyllithium (2.1 $\mathrm{M}$ in hexanes; $24 \mathrm{ml}, 50.1 \mathrm{mmol}$ ) was added to a chilled $\left(0^{\circ} \mathrm{C}\right)$ suspension of cholesten-3 $\beta$-ol $(20.0 \mathrm{~g}, 51.7 \mathrm{mmol})$ in $n$-hexane $(80 \mathrm{ml})$. The resulting solution was gradually warmed to $25^{\circ} \mathrm{C}$, and then treated with freshly distilled chloral $(5.0 \mathrm{ml}, 51.3 \mathrm{mmol})$. After stirring for $15 \mathrm{~min}$, the reaction mixture was quenched with acetic anhydride $(10 \mathrm{ml}, 106 \mathrm{mmol})$. The resulting tan slurry was dissolved in benzene $(75 \mathrm{ml})$ and extracted with $0.1 \mathrm{~N}$ sodium bicarbonate $(3 \times 50 \mathrm{ml})$, water $(5 \times 50 \mathrm{ml})$, and brine $(50 \mathrm{ml})$. The organic phase was separated, dried over anhydrous magnesium sulfate and then concentrated in vacuo to afford a viscous tan oil which slowly crystallized on standing. The crude product was recrystallized from warm absolute ethanol to give, after drying, $22.3 \mathrm{~g}(75 \%)$ of 6 as an off-white crystalline solid; mp 104-106 ${ }^{\circ} \mathrm{C} ;[\alpha]_{\mathrm{D}}^{R T}=(-) 13.2^{\circ}(1.0 \mathrm{~g} /$ $\left.2.0 \mathrm{ml} \mathrm{CHCl}{ }_{3}\right)$; IR $\left(\mathrm{CHCl}_{3}\right): 1750 \mathrm{~cm}^{-1}(\mathrm{C}=\mathrm{O}$ stretch); ${ }^{13} \mathrm{C}$ NMR $\left(\mathrm{CDCl}_{3}\right): \partial 170.8,140.9$, $123.7,98.3,97.9,82.7,82.0,56.8,56.2,50.5$, $42.9,42.4,40.0,39.6,37.7,36.8,36.4,36.0$, $32.5,32.1,28.5,28.1,24.6,24.4,24.0,22.8$, 22.6, 21.2, 19.5, 18.8, 12.2 ppm; Anal. Calcd for $\mathrm{C}_{31} \mathrm{H}_{49} \mathrm{O}_{3} \mathrm{Cl}_{3}: \mathrm{C}, 64.63 \% ; \mathrm{H}, 8.57 \% ; \mathrm{Cl}$, $18.46 \%$. Found: C, $64.64 \%$; H, 8.65\%; Cl, $18.33 \%$.

\section{General Procedures}

Melting points were determined in open capillary tubes with a Laboratory Devices Mel-Temp unit and are uncorrected. ${ }^{13} \mathrm{C}$ NMR spectra were obtained at $22.6 \mathrm{MHz}$ on a Varian CFT-20 spectrometer with complete proton decoupling. Infrared spectra were recorded on a Perkin-Elmer 727 spectrometer. Samples were typically measured in solution. Analytical data were furnished by the University of Massachusetts (Amherst) Microanalytical Laboratory. Glass sheet-casting assemblies used for the fabrication of polychloral sheets were prepared by sandwiching a 3500 denier polyurethane thread between two $18 \mathrm{~cm} \times 18 \mathrm{~cm}$ pyrex glass plates. When clamped firmly together, the plates were separated by a gap of 0.1 to $0.2 \mathrm{~mm}$. Initiated monomer solutions were syringed into the void separating the two plates immediately prior to the polymerization step. A more detailed description of this apparatus is provided elsewhere. ${ }^{26}$

\section{Chiroptical Studies}

Optical rotation measurements were carried out on a Perkin-Elmer 141 MC polarimeter using wavelengths available with standard sodium and mercury lamps. Soluble compounds were measured in $1.0 \mathrm{dm}$ solution cells thermostated to $20.0^{\circ} \mathrm{C}$. The polymer, lacking solubility in organic and aqueous solvent media, was evaluated at ambient temperatures in the solid state. ${ }^{28}$ Each polychloral disk, gently sandwiched between two optically transparent glass plates, was positioned directly 
into the polarimeter cavity. Optical rotation, $\alpha$, was measured at three distinct orientations, $120^{\circ}$ apart, by carefully rotating the disk in the plane perpendicular to the light beam. If the average of the three rotation measurements had a standard deviation $\geq 15 \%$, the disk was judged to be optically anisotropic and not suitable for further study. A total of six to eight isotropic polymer samples were evaluated in this way for each condition under which polychloral was prepared. Rotation data, reported here as specific rotations, $[\alpha]_{\lambda}^{R T}$ (in $\operatorname{deg} \mathrm{dm}^{-1} \mathrm{~g}^{-1} \mathrm{~cm}^{3}$ ), were calculated using the following expression:

$$
[\alpha]_{\lambda}^{R T}=(\alpha)(1 / L)(1 / \rho)
$$

where $\alpha$ is the average rotation at wavelength $\lambda, L$ is sample thickness in $d m$ and $\rho$ is the density of polychloral $\left(1.90 \mathrm{~g} \mathrm{~cm}^{-3}\right) .{ }^{13}$ All specific rotation data were obtained at room temperature $(R T)$ and are typically $\pm 3-5 \%$.

\section{RESULTS}

Polymer samples suitable for solid state chiroptical measurements were prepared using a multi-step cryotachensic polymerization process outlined in Scheme 1. Alkoxide initiators were first homogeneously dispersed in chloral monomer at elevated temperatures. After a desired holding or incubation period, polymerization was induced by rapidly cooling the initiated monomer solutions below their ceiling $\left(T_{\mathrm{c}}\right)$ temperatures. When prepared in thin sheet or disk form, the semicrystalline polymer possessed considerable mechanical strength and high levels of optical isotropy and transparency after acid stabilization and diphenyl ether post-treatment. Polychloral

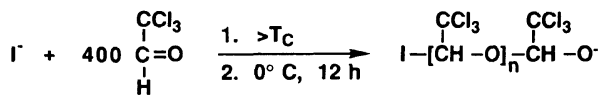

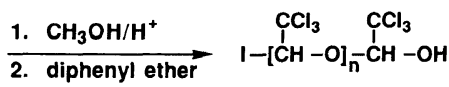

Scheme 1. samples prepared with the achiral initiator lithium $t$-butoxide were optically inactive within the limits of experimental error and these served as a control for subsequent chiroptical measurements.

\section{Polymerization of Chloral with Lithium $R(-)$ - and $S(+)-2-O c t a n o x i d e s$}

Initiators $R(-)-1$ and $S(+)-1$ were prepared from their corresponding secondary alcohols with 0.98 equiv of $n$-butyllithium. A slight excess of each alcohol was used to limit the presence of any achiral initiating species in the polymerization reaction. In stark contrast to the lithium $t$-butoxide case, the polymerization of chloral (method I) with the chiral octanoxides afforded a polymer with exceptionally high rotation magnitudes. As illustrated in Figure 1, the optical activity imparted to polychloral was directly influenced by the absolute configuration of the 2-octanoxide, and, the magnitude of the rotation enhancements was related to the holding temperatures employed prior to the formal polymerization step. Specifically, at the $65^{\circ} \mathrm{C}$ holding isotherm, initiator $R(-)-1$ afforded a polymer with a $[\alpha]_{\mathrm{D}}^{R T}$ of $(+) 4280^{\circ} \pm 190^{\circ}$ while its mirror $S(+)$ antipode furnished a sample with $[\alpha]_{\mathrm{D}}^{R T}=$ $(-) 3890^{\circ} \pm 200^{\circ}$. At the $85^{\circ} \mathrm{C}$ isotherm, rotation magnitudes in polychloral were signifi-

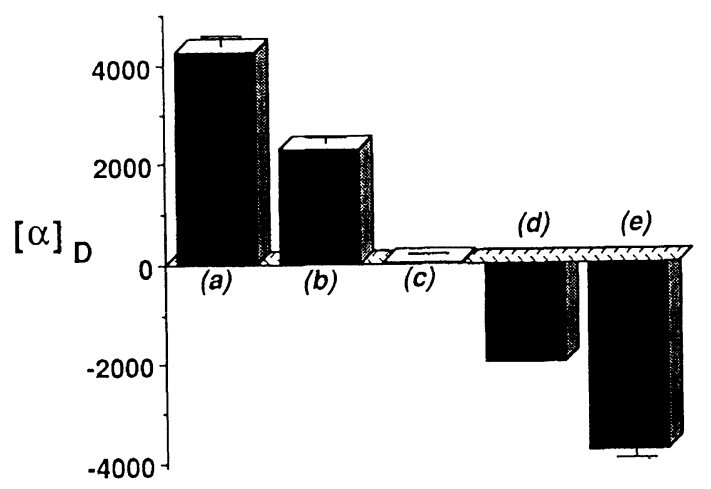

Figure 1. Specific rotation of polychloral prepared with: (a) $R(-)-1$ at $65^{\circ} \mathrm{C}$; (b) $R(-)-1$ at $85^{\circ} \mathrm{C}$; (c) rac-1 at 65 or $85^{\circ} \mathrm{C}$; (d) $S(+)-1$ at $85^{\circ} \mathrm{C}$ and (e) $S(+)-1$ at $65^{\circ} \mathrm{C}$ (method $\mathrm{I}$ : holding time $=10 \mathrm{~min}$ ). 
cantly reduced, with $[\alpha]_{\mathrm{D}}^{R T}$ values dropping to near $(+)$ or $(-) 2000^{\circ}$, respectively. Consistent with these observations, polychloral prepared with the racemic octanoxide was optically inactive, with a measured specific rotation of $(+) 7^{\circ} \pm 16^{\circ}$.

The influence of holding temperature on the polymerization process was probed in greater detail for initiator $R(-)$-1. As can be noted in Figure 2 (upper curve), specific rotations observed in the polymer scaled inversely with holding temperature, with a $60 \%$ drop in rotation magnitudes occurring over the $30^{\circ}$ temperature range studied. Increasing the length of time between the initiation and polymerization events beyond the standard 10 minute interval (polymerization method II) also brought about concomitant reductions in op-

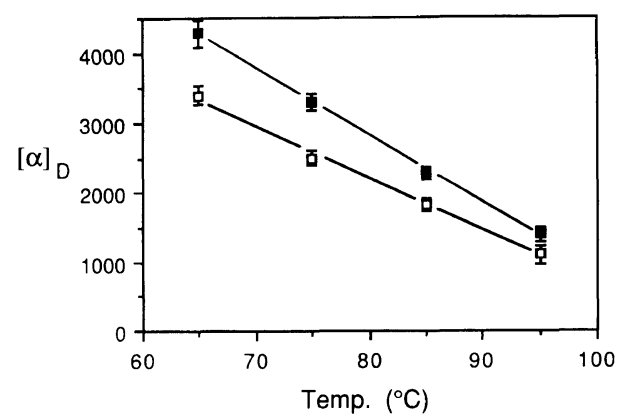

Figure 2. Specific rotation of polychloral prepared with $R(-)-1[\square]$ or with $2[\square]$ as a function of holding temperature prior to the polymerization step (method I: holding time $=10 \mathrm{~min}$ ).

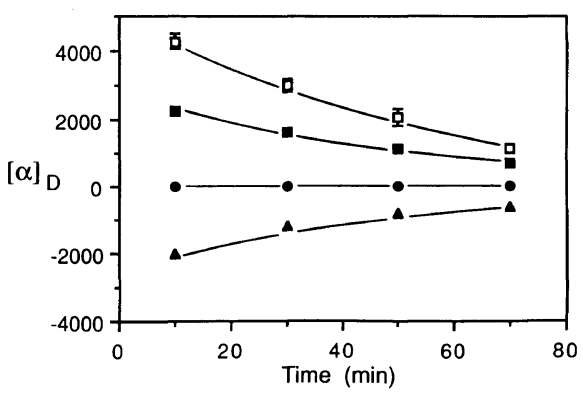

Figure 3. Specific rotation of polychloral prepared with $R(-)-1$ at $65^{\circ}[\square]$ and $85^{\circ} \mathrm{C}[\mathbf{\square}], \mathrm{cac}-1$ at $85^{\circ} \mathrm{C}[\mathbf{O}]$ and $S(+)-1$ at $85^{\circ} \mathrm{C}[\mathbf{\Delta}]$ as a function of time between initiation and polymerization steps (method II). tical rotatory power. For both the $R(-)$ and $S(+)$ initiating species at the $65^{\circ}$ and the $85^{\circ} \mathrm{C}$ holding isotherms, the rotation magnitudes ultimately observed in polychloral were noted to fall sharply for holding periods in excess of 10 minutes (Figure 3). Indeed, inducing polymerization after incubating the initiated monomer solutions for periods exceeding one hour invariably afforded polymer samples with little or no solid state optical rotations. As expected, alterations in holding time or temperature had a negligible effect on polychloral fabricated with the racemic 2-octanoxide. In all cases, polymer prepared with rac-1 was devoid of any measurable optical activity.

\section{Polymerization of Chloral with Sterol Lithium Alkoxides}

Chiral initiators 2-5 were derived from commercially available $\mathrm{C}_{27}$ monohydroxy sterols in a manner analogous to the preparation of the 2-octanoxides detailed above. Polychloral prepared with 2-5 (method I) exhibited large dextrorotatory rotations when evaluated at the sodium D-line. Notably, at the $85^{\circ} \mathrm{C}$ isotherm, the alkoxides of 5-cholesten-3 $\beta$-ol (2), cholestan-3 $\beta$-ol (3) and copros$\tan -3 \alpha$-ol (5) all behaved similarly, providing polychloral samples with specific rotations near $(+) 1900^{\circ}$ (Figure 4). In contrast, lithium coprostan-3 $\beta$-oxide (4) afforded

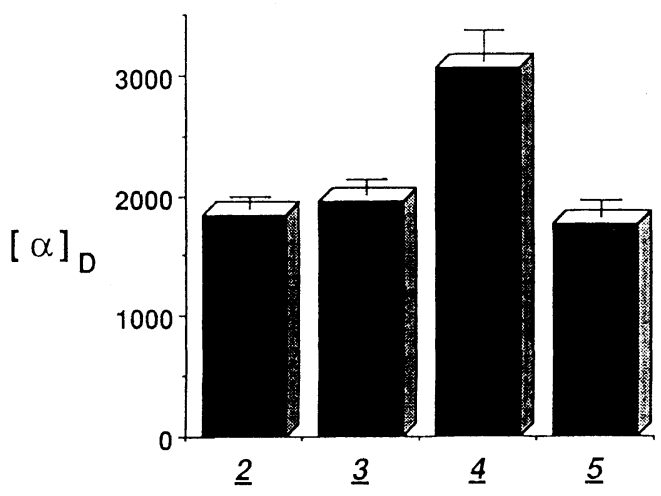

Figure 4. Specific rotation of polychloral prepared with sterol alkoxide initiators $\mathbf{2}-\mathbf{5}$ at $85^{\circ} \mathrm{C}$ (method I: holding time $=10 \mathrm{~min}$ ). 


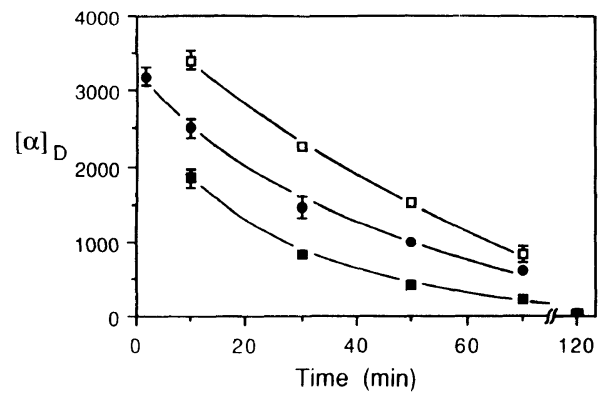

Figure 5. Specific rotation of polychloral prepared with 2 at $65^{\circ}[\square], 75^{\circ}[O]$ and $85^{\circ} \mathrm{C}[\square]$ as a function of time between initiation and polymerization steps (method II).

a polymer with a $[\alpha]_{\mathrm{D}}^{R T}$ value of $(+) 2970^{\circ} \pm$ $300^{\circ}$ under the same set of experimental conditions. Like the 2-octanoxide case above, the chiroptical properties in the polymer were also sensitive to the manner in which the polymerizations were carried out. The magnitude of the rotation values imparted to polychloral by alkoxide 2 was strongly coupled to both the holding time and temperature employed prior to the formal polymerization step. As depicted graphically in Figure 2 and Figure 5, higher holding temperatures and longer holding periods again resulted in significantly diminished rotation values in the polychloral samples. Similar trends were observed for the cholestan-3 $\beta$-oxide initiator (Figure 6) and for the two coprostanoxide initiators $\mathbf{4}$ and $\mathbf{5}$ (data not shown).

Synthesis and Chiroptical Evaluation of an Asymmetric End Group Mimic

Also examined were the chiroptical properties of 2,2,2-trichloro-1-[(3 $\beta)$-cholest-5-en3-yloxy]ethyl acetate (6), a low molecularweight model compound designed to approximate asymmetric endgroup structure in the polymer chain. The steroid derivative was synthesized as a mixture of diastereomers in $75 \%$ overall yield by treating 2 with a single equivalent of chloral monomer, followed by endcapping with acetic anhydride (Scheme 2). At the sodium D-line, this end group mimic possessed a specific rotation of $(-) 13.2^{\circ}$ in

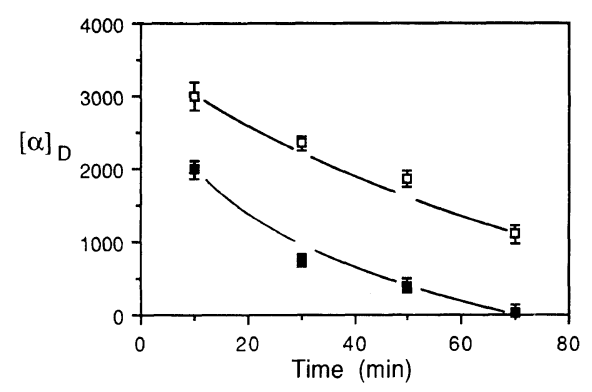

Figure 6. Specific rotation of polychloral prepared with 3 at $65^{\circ}[\square]$ and $85^{\circ} \mathrm{C}[\square]$ as a function of time between initiation and polymerization steps (method II).

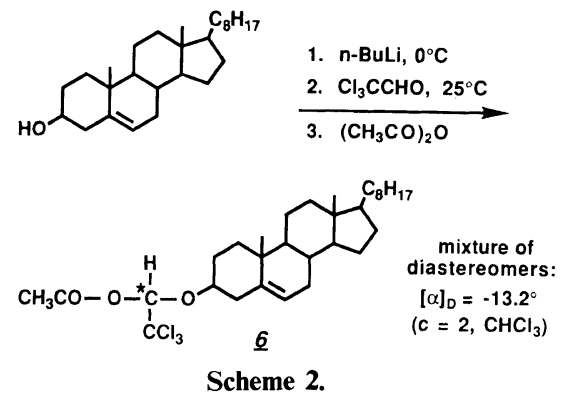

$\mathrm{CHCl}_{3}$. For comparison, the specific rotation of the parent 5 -cholesten- $3 \beta$-ol was observed to be near (-) $39^{\circ}$ when measured under the same conditions. With some interest, it was noted that higher oligomeric products were formed when a five-fold excess of chloral monomer was employed in the reaction sequence. Because of difficulties encountered in purifying the complex product mixture, however, the stereochemistry and chiroptical properties of these higher oligomeric adducts were not investigated further.

\section{ORD Analysis of Optically Active Polychloral}

The optical rotatory dispersion (ORD) characteristics of polychloral prepared in this study were evaluated in some detail. Two optically isotropic polymer samples obtained with initiators $R(-)-1\left([\alpha]_{\mathrm{D}}^{R T}=(+) 2680^{\circ}\right)$ and $2\left([\alpha]_{\mathrm{D}}^{\bar{R} \bar{T}}=(+) 2650^{\circ}\right)$ were selected for this purpose. Using the $\mathrm{Na}$ and $\mathrm{Hg}$ lines available on the Perkin-Elmer 141 MC polarimeter, the plain ORD curves provided in Figure 7 were 


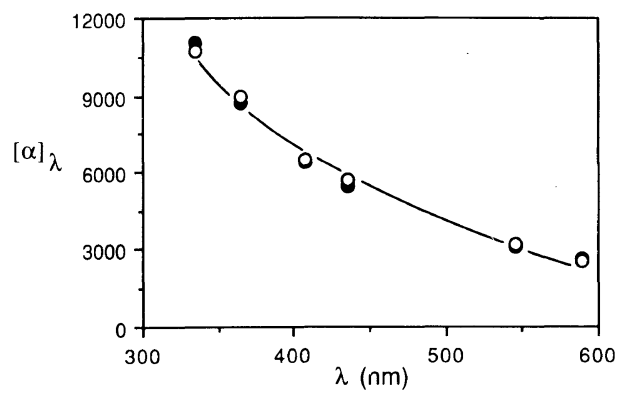

Figure 7. ORD curves for optically active polychloral prepared with $R(-)-\mathbf{1}$ [O] and 2 [O].

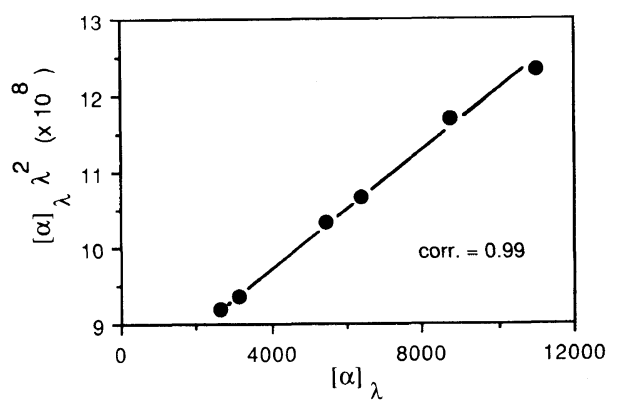

Figure 8. Single-term Drude plot for optically active polychloral prepared with 2 .

constructed. As is clearly evident, the data are characterized by a monotonic increase in rotation magnitudes at shorter wavelengths, with specific rotations in both cases reaching (+) $11000^{\circ}$ at the $334 \mathrm{~nm} \mathrm{Hg}$-line.

Further manipulation of the data suggested that the dispersion curves could be fitted to a single-term Drude ${ }^{29}$ equation:

$$
[\alpha]_{\lambda} \lambda^{2}=[\alpha]_{\lambda} \lambda_{\mathrm{c}}^{2}+A
$$

where $[\alpha]_{\lambda}$ is the specific rotation of the polymer at wavelength $\lambda, \lambda_{\mathrm{c}}$ is the wavelength of the dominant optical transition, and $A$ a constant unique to the system under investigation. For polychloral initiated with $\mathbf{2}$, a plot of $[\alpha]_{\lambda} \lambda^{2}$ versus $[\alpha]_{\lambda}$ afforded a straight line with a correlation coefficient of 0.99 (Figure 8 ). From the slope of the line, the dispersion constant, $\lambda_{\mathrm{c}}$, was calculated to be near $193 \mathrm{~nm}$. Polychloral prepared with the $R(-)-1$ initiator afforded strikingly similar results, giving a calculated dispersion constant of $197 \mathrm{~nm}$.

\section{DISCUSSION}

Helix Sense-Selective Polymerization of Chloral

Polychloral is an isotactic, semicrystalline macromolecule with $4_{1}$ helical order in its solid state. The polymer can be readily prepared with a variety of anionic initiators and has been the subject of a number of detailed studies over the past twenty years. ${ }^{5,6}$ Preliminary efforts in our laboratory with a number of chiral initiators have hinted that the highest optical rotations in polychloral are obtained when strong, sterically demanding nucleophiles are employed as initiating species. ${ }^{7}$ Weak initiators, or those with little steric bulk centered about their anionic sites, have generally provided smaller rotation magnitudes. ${ }^{8}$ In the present study, chiral lithium alkoxides derived from commercially available secondary alcohols were used to impart exceptionally large solid state rotation magnitudes into the polymer samples.

Focusing initially on the structurally less complex octanoxide initiators, the rotation data presented in Figures $1-3$ suggest that (i) the optical activity imparted to polychloral is directly influenced by the absolute configuration of the 2-octanoxide, and (ii) the magnitude of the rotations is inversely related to both the holding time and temperature employed prior to the formal polymerization step. Specifically, opposite initiator configurations did lead to nearly opposite optical rotations, with racemic initiator yielding an optically inactive polymer. Furthermore, higher holding temperatures and longer holding times consistently resulted in lower rotation values in the polychloral samples.

These trends can be rationalized by considering the effects of initiator configuration, holding time and temperature on the embryonic stages of helix development prior to the formal polymerization step. As depicted in Scheme 3, the chiral octanoxide $\left({ }^{*} \mathrm{I}^{-}\right)$can approach prochiral monomer $\left(\mathbf{M}_{1}\right)$ in one of two stereochemically distinct ways, i.e., from 


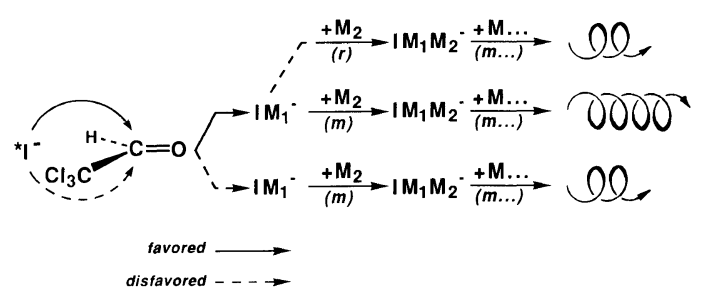

Scheme 3.

above or below the plane of the monomer's carbonyl moiety. Hypothetically, if the $R$ enantiomer of the octanoxide favors one route of attack, then its mirror $S$-isomer should favor the opposite route with equal frequency. For a given mode of attack, subsequent oligomerization steps in the holding solution $\left(+\mathbf{M}_{2}\right.$, $\mathrm{M} \cdot \cdots)$ via a series of energetically favored meso (m) placements would then yield embryonic helices directed predominantly into either a right or left screw sense. At this stage in the chain growth process, an odd number of racemic $(r)$ growth steps would act to invert the orientation of the nascent helical screws. On inducing polymerization by cooling the solution below its ceiling temperature, a rapid series of highly favored meso placements would yield optically active, isotactic polymer, where the magnitude of its optical rotation is a function of the number of excess helical coils which lie in the predominant screw direction. In the present scenario, raising the temperature of the holding solution from $65^{\circ} \mathrm{C}$ to $85^{\circ} \mathrm{C}$ would most likely result in a loss of stereoselectivity for the attacking initiator or oligomer alkoxide. Furthermore, incubating the initiated monomer solutions for longer time periods could enhance racemization or rearrangement processes centered at the terminal alkoxide. In either case, the net effect would be a loss of helical asymmetry, and thus optical activity, in the polymer sample ultimately prepared.

This scheme is fully consistent with a number of theoretical and experimental studies. Molecular mechanics (MM2) calculations for polychloral and several of its oligomeric model compounds ${ }^{30}$ have predicted that, following attack of the initiating alkoxide, oligomeric chain growth in the initiated monomer solution becomes highly stereoselective for meso addition as the trimer stage is reached. Additional meso placements then quickly generate the first turn in the polymer's emerging $4_{1}$ helical screw. At this point, energetically disfavored racemic placements are suppressed along the propagating polyaldehyde chain. Experimental evidence in support of these predictions has been gained from the examination of a series of acetate endcapped chloral oligomers, prepared with the lithium salt of (-)-borneol under conditions similar to those employed here for the polymerization reaction. The analysis of chloral unimer, dimer, trimer, tetramer and pentamer species by high pressure liquid chromatography, X-ray crystallography, and ${ }^{1} \mathrm{H},{ }^{13} \mathrm{C}$ and $2 \mathrm{D}$ NMR spectroscopies has shown that the higher oligomeric adducts possess well defined stereo-structures, with individual monomer units coupled together in meso fashion. ${ }^{19-22}$ Indeed, the tetramer and pentamer derivatives exhibit solid state conformations which are similar to the $4_{1}$ helical geometry found in isotactic polychloral. Predictably, the occurrence of random racemic placements in these model compounds was found to be greater with the use of higher reaction temperatures as was the formation of a number of undesired side products. Similar findings have been observed for chloral oligomers prepared with the achiral initiator lithium $t$-butoxide. ${ }^{17,18}$

Armed with some understanding of the 2-octanoxide data, attention can be directed to the structurally more complex steroid initiators 2-5. As drawn above, each of the conformationally restricted alkoxides is comprised of three fused cyclohexane rings (A, B, C), a terminal cyclopentane ring (D) and a pendent aliphatic chain at C. ${ }^{17}$ Steric and electronic modifications near the initiators' reactive sites include $\beta$-versus $\alpha$-orientation of the alkoxide moiety, saturation versus unsaturation at 
$\mathrm{C}^{5}-\mathrm{C}^{6}$ and trans versus cis $\mathrm{A} / \mathrm{B}$ ring juncture.

Polychloral samples prepared with 2-5 possessed large dextrorotatory rotations when evaluated at the sodium D-line. With some surprise, no levorotatory polymer was obtained. Moreover, initiator 4, with its axial alkoxide orientation, afforded rotation magnitudes in the polymer that were some $60 \%$ higher than those realized under identical conditions with the other steroid initiating species (Figure 4). These results are consistent with expectations derived from Corey-PaulingKolton molecular modelling efforts which suggest that the more sterically congested coprostan- $3 \beta$-oxide should possess a higher level of stereoselectivity for the initiation step as compared to $\mathbf{2 , 3}$, or $\mathbf{5}$. Indeed, our models show that the steroid framework in 2,3 , and 5 is actually directed away from the intiating site at $\mathrm{C}^{3}$ and is probably unable to render assistance in orienting monomer units undergoing nucleophilic attack.

In good agreement with the 2-octanoxide data described earlier, rotation magnitudes in the polymer samples were observed to fall sharply when higher holding temperatures (Figure 2) and longer holding times (Figures 5 and 6) were utilized to effect the cryotachensic polymerization sequence. Interestingly, this drop in optical rotatory power was observed for all four steroid initiating systems and, to a first approximation, was independent of initiator structure. Again, as proposed in Scheme 3 above, more rigorous polymerization conditions probably foster competing racemization or rearrangement processes which, alone or in concert, effectively diminish the level of helical asymmetry in the polymer sample.

The results of the present study, when taken in conjunction with several earlier findings, ${ }^{6-8}$ strongly support the notion that maximum optical rotations in polychloral will be realized when sterically demanding alkoxide initiators and relatively mild conditions are employed to effect the polymerization reaction. As the trends in Figures $1-6$ suggest, the preparation of polychloral solids with specific rotation magnitudes exceeding $5000^{\circ}$ may be feasible using this approach. Of perhaps greater significance, the data obtained here clearly demonstrate that a proper choice of initiator configuration, coupled with the controlled manipulation of the cryotachensic polymerization process, provides an effective means for tuning or adjusting the optical activity in polychloral. Indeed, in our hands, polymer solids with specific rotations ranging from $(+)$ $4000^{\circ}$ to $(-) 4000^{\circ}$ were routinely, and reproducibly, fabricated with this technique.

The magnitude of the optical rotatory power incorporated into polychloral also merits some comment. It is interesting to note that the rotation enhancements observed here greatly exceed those attained for other helical macromolecules prepared by similar methodology. For example, helical polymers of triphenylmethyl methacrylate ${ }^{9,10}$ and several substituted isocyanides ${ }^{11,12}$ exhibit maximum $[\alpha]_{\mathrm{D}}$ values which are generally one to two orders of magnitude smaller than those realized in the present study. While the origins of this difference remain to be fully elucidated, it should be mentioned that in these latter cases, chiroptical measurements were carried out in solution and not in the solid state where some degree of molecular ordering is possible. While every effort was made to prepare and measure only isotropic samples of polychloral (vide supra), an intriguing possibility remains that bundles of closely packed helices present in crystalline regions of the polymer could give rise to a mode of orientation which, by coincidence, is aligned with the direction of polarimetric observation. As detailed by Bonsignori and Lorenzi, ${ }^{28}$ this type of orientation, if present, would be difficult to detect by chiroptical methods and could enhance the rotation magnitudes recorded in the present study. Thus far, attempts to prepare agitated, statistically isotropic suspensions of finely powdered polychloral suitable for chiroptical measurements have been thwarted 
by the polymer's intractable nature. Suspension measurements, if technically feasible, would provide an interesting check on this possibility.

\section{Asymmetric Endgroup Mimic}

Although structurally devoid of main chain or pendent side chain chiral centers, polychloral prepared with asymmetric initiating alkoxides contains a number of chiral endgroups which contribute to observed optical rotation behavior. As part of this study, it was desired to synthesize a stable chloral addition product which could serve as a low molecular weight model compound approximating asymmetric endgroup structure in the polymer samples. One such derivative, 2,2,2-trichloro-1-[(3 $\beta)$-cholest-5-en-3-yloxy]ethyl acetate (6), was efficiently prepared from initiator 2 under conditions similar to those employed in the polymerization reaction (Scheme 2). As expected, 6 was isolated as a mixture of diastereomers due to the presence of both $R$ - and $S$-configurations at its acetal carbon moiety. Analysis of the crude product by NMR spectroscopy showed clearly that the two isomers were present in unequal amounts consistent with the proposed mechanism for the asymmetric polymerization reaction. However, no attempt was made to resolve the product into its individual $R$ and $S$ components. As the sodium D-line, 6 exhibited a specific rotation of $(-) 13.2^{\circ}$ in chloroform, a value that is opposite in sign and several orders of magnitude smaller than the strong dextrorotatory rotations observed for polychloral initiated with 2 . This result is in excellent agreement with other chloral endgroup ${ }^{7}$ and chloral oligomer studies ${ }^{22}$ and confirms that chiral initiator fragments in the polymer, even if present at high relative concentrations, contribute little to the large rotation magnitudes reported here.

\section{ORD Studies}

Additional insight into the nature of asymmetry present in polychloral was gained by carrying out optical rotatory dispersion (ORD) analyses using the $\mathrm{Hg}$ - and $\mathrm{Na}$-lines available on a standard polarimeter. When evaluated between 334 and $589 \mathrm{~nm}$, polymer samples prepared with initiators $R(-)-1$ and 2 afforded plain (normal) ORD curves which were free of any inflections or extrema (Figure 7). Consistent with earlier investigations in our laboratory, ${ }^{7}$ the optical rotatory dispersions were found to fit the simple Drude ${ }^{29}$ equation (eq 2), with calculated dispersion constants falling between 193 and $197 \mathrm{~nm}$ (Figure 8). To a first approximation, these data suggest that the major electronic transition within polychloral lies below $200 \mathrm{~nm}$, far removed from the $589 \mathrm{~nm}$ sodium D-line where optical rotation measurements were routinely gathered. Unfortunately, attempts to probe this transition directly were limited by the spectral range of the polarimeter. That the calculated dispersion constants show little dependence on initiator structure provides added evidence that the optical rotations observed in polychloral are derived primarily from the polymer's secondary structure and not from chiral fragments that are localized at the terminus of each polyaldehyde chain.

\section{CONCLUDING REMARKS}

Chiral alkoxide initiators derived from commercially available secondary alcohols were used to impart large solid state rotation magnitudes into polychloral via a helix-sense selective polymerization process. The sign and magnitude of the optical rotations were found to depend on both initiator structure and the conditions under which the polymerizations were carried out. By carefully manipulating the polymerization reaction, polychloral samples with $[\alpha]_{\mathrm{D}}$ values ranging from $(+) 4000^{\circ}$ to (-) $4000^{\circ}$ were routinely prepared. Model end-group studies confirmed that the presence of asymmetric initiator fragments in the polymer contributed little to the observed 
rotation magnitudes reported here. As expected, polychloral prepared with achiral or racemic initiators was completely devoid of any measurable optical rotatory power.

Currently, we are expanding the scope of our initiator investigations to include a number of chiral, tertiary alkoxides in an effort to maximize the rotation magnitudes imparted to polychloral. Also underway are a new generation of chloral oligomer studies aimed at assigning absolute helical screw directions to dextrorotatory and levorotatory polymer samples. Finally, it has not escaped our attention that it may be possible to manipulate the cryotachensic polymerization process in such a way as to unidirectionally align the embryonic helical oligomers in solution before or during the onset of polymerization. This process could potentially give rise to a highly anisotropic, three dimensional polymer solid with directionally dependent chiroptical, chemical and physical behavior. We anticipate that polychloral fabricated in this manner would possess novel properties that could be exploited in a variety of solid-state applications.

Acknowledgment. This work, Part 42 in the series Haloaldehyde Polymers, was supported, in part, by grants from the National Science Foundation (DMR-80-17058 and DMR-8302134) and the Materials Research Laboratory of the University of Massachusetts. The authors would like to thank Dr. W. J. Harris (Dow Chemical) and Professor W. H. Stockmayer (Dartmouth) for a number of helpful and enlightening discussions and Professor K. Hatada (Osaka University) for his continued assistance with the chloral oligomer and X-ray crystallographic studies.

\section{REFERENCES}

1. O.Vogl and G. D. Jaycox, Polymer, 28, 2179(1987).

2. Y. H. Kim and O. W. Webster, J. Am. Chem. Soc., 112, 4592 (1990).
3. C. J. Hawker and J. M. J. Frechet, J. Am. Chem. Soc., 112, 7638 (1990).

4. D. A. Tomalia, A. M. Naylor, and W. A. Goddard, Angew. Chem., 29, 139 (1990).

5. L. S. Corley, G. D. Jaycox, and O. Vogl, J. Macromol. Sci.-Chem., A25, 519 (1988) and references cited therein.

6. L. S. Corley and O. Vogl, Polym. Bull., 3, 211 (1980).

7. W. J. Harris and O. Vogl, J. Macromol. Sci.-Chem., A26, 1067 (1989).

8. W. J. Harris and O. Vogl, J. Macromol. Sci.-Chem., A26, 1083 (1989).

9. Y. Okamoto, K. Suzuki, K. Ohta, K. Hatada, and H. Yuki, J. Am. Chem. Soc., 101, 4763 (1979).

10. Y. Okamoto, E. Yashima, T. Nakano, and K. Hatada, Chem. Lett., 759 (1987).

11. R. J. M. Nolte, A. J. M. van Beijnen, and W. Drenth, J. Am. Chem. Soc., 96, 5932 (1974).

12. W. Drenth and R. J. M. Nolte, Acc. Chem. Res., 12, 30 (1979).

13. P. Kubisa, L. S. Corley, T. Kondo, M. Jacovic, and O. Vogl, Polym. Eng. Sci., 21, 829 (1981).

14. E. G. Brame, A. M. Raevsky, G. K. Semin, G. D. Jaycox, and O. Vogl, Polym. Bull., 10, 521 (1983).

15. W. J. Harris, J. R. Haven, J. L. Koenig, and O. Vogl, Makromol. Chem., 184, 1234 (1983).

16. A. Novak and E. Whalley, Trans. Faraday Soc., 55, 1490 (1959).

17. J. Zhang, G. D. Jaycox, and O. Vogl, Polymer, 29, 707 (1988).

18. G. D. Jaycox, F. Xi, O. Vogl, K. Hatada, K. Ute, and T. Nishimura, Polym. Prepr., Am. Chem. Soc., Div. Polym. Chem., 30(2), 167 (1989).

19. J. Zhang, G. D. Jaycox, and O. Vogl, Polym. J., 19, 606 (1987).

20. G. D. Jaycox, K. Hatada, F. Xi, and O. Vogl, Pacific Polym. Prepr., 1, 267 (1989).

21. K. Ute, K. Oka, M. Kashiyama, K. Hatada, F. Xi, and O. Vogl, Makromol. Chem., in press.

22. O. Vogl, G. D. Jaycox, W. J. Simonsick, Jr., and K. Hatada, J. Macromol. Sci.-Chem., submitted.

23. G. D. Jaycox and O. Vogl, Makromol. Chem., Rapid Commun., 11, 61 (1990).

24. G. D. Jaycox and O. Vogl, Polym. Commun., 30, 354 (1989).

25. M. R. Winkle, J. M. Lansinger, and R. C. Ronald, J. Chem. Soc., Chem. Commun., 88 (1980).

26. G. D. Jaycox, M. S. Thesis, University of Massachusetts (Amherst), 1984.

27. O. Vogl, H. C. Miller, and W. H. Sharkey, Macromolecules, 5, 658 (1972).

28. O. Bonsignori and G. P. Lorenzi, J. Polym. Sci., A-2, 8, 1639 (1970).

29. P. Drude, "Lehrbuch der Optik," 2nd ed, S. Hirzel Verlag, Leipzig, 1900.

30. A. Abe, K. Tasaki, K. Inomata, and O. Vogl, Macromolecules, 19, 2707 (1986). 